\title{
Cardiac and hepatic phenotype of diabetes in the presence and the absence of obesity - mechanistic role of ectopic/visceral adiposity
}

\author{
Eylem Levelt ${ }^{1,4^{*}}$, Michael Pavlides ${ }^{1}$, Masliza Mahmod ${ }^{1}$, Catherine Kelly ${ }^{2}$, Joanne Sellwood ${ }^{1}$, Sheena Thomas ${ }^{3}$, \\ Jane M Francis ${ }^{1}$, Jurgen E Schneider ${ }^{1}$, Chris Rodgers ${ }^{1}$, William T Clarke ${ }^{1}$, Nikant Sabharwal ${ }^{3}$, \\ Charalambos Antoniades ${ }^{3}$, Kieran Clarke ${ }^{4}$, Theodoros D Karamitsos ${ }^{1}$, Oliver Rider ${ }^{1}$, Stefan Neubauer ${ }^{1}$
}

From 19th Annual SCMR Scientific Sessions

Los Angeles, CA, USA. 27-30 January 2016

\section{Background}

Ectopic/visceral adiposity is frequently observed in type 2 diabetes mellitus (T2DM) and is linked to cardiovascular mortality. Non-alcoholic fatty liver disease (NAFLD) is an example of ectopic fat accumulation in a visceral organ, also strongly linked with cardiovascular mortality. We assessed the diabetes-associated cardiac and hepatic changes, and hypothesised that they will be amplified by the co-existence of obesity, and that the ectopic adiposity may play a pathophysiological role in the cardiac and hepatic phenotype of diabetes.

\section{Methods}

Twenty-seven obese T2DM (O-T2DM) patients, fifteen lean T2DM (L-T2DM) patients, and twelve healthy volunteers were studied. T2DM patients underwent cardiac CT (epicardial fat quantification and exclusion of significant $\mathrm{CAD}$ ), cardiac MRI (cine and tagging), ${ }^{1} \mathrm{H}$-, ${ }^{31} \mathrm{P}$-MRS for myocardial triglyceride (MTG) and $\mathrm{PCr} /$ ATP respectively, and a multi-parametric liver MRI scan, including ${ }^{1} \mathrm{H}$-MRS for hepatic triglyceride (HTG), $\mathrm{T} 1$ and $\mathrm{T} 2 *$ mapping yielding an 'iron-corrected T1' (cT1), a parameter which allows non-invasive quantification of fibroinflammatory liver disease. Healthy volunteers underwent identical MRI protocols.

\section{Results}

Demographic, biochemical and multiparametric MRI results are provided in Figure 1.

'OCMR, University of Oxford, Oxford, United Kingdom

Full list of author information is available at the end of the article
When comparing L-T2DM to controls, diabetes, even in the absence of obesity, was associated with increased LV mass $(p=0.03)$, impaired myocardial energetics $(\mathrm{p}=0.04)$, increased MTG $(\mathrm{p}=0.01)$ and HTG $(\mathrm{p}=0.04)$. While cardiac structural changes, and abnormalities in MTG and PCr/ATP were similar between the two T2DM groups, epicardial fat volumes $(\mathrm{p}=0.04)$ and HTG $(\mathrm{p}=0.01)$ were significantly increased in O-T2DM patients compared to L-T2DM. Moreover, HTG and epicardial fat volumes correlated negatively with the peak systolic circumferential strain and diastolic strain rates (Figure 2 for all), and in line with this, these functional changes were only impaired in O-T2DM patients ( $\mathrm{p}<0.001$ and $\mathrm{p}=0.006$ respectively compared to controls), supporting a potential mechanistic role of ectopic adiposity for cardiac dysfunction in T2DM. MTG did not correlate with HTG or epicardial fat volumes. Finally, fibroinflammatory liver disease (elevated cT1) was also only evident in O-T2DM $(\mathrm{p}=0.004$ and $\mathrm{p}<$ $0.001 \mathrm{vs}$ L-T2DM patients and controls, respectively), and liver cT1 also correlated with HTG and epicardial fat volumes $(\mathrm{p}<0.001$ and $\mathrm{p}=0.01$ respectively).

\section{Conclusions}

We demonstrate here, for the first time, not only that ectopic adiposity is more pronounced in obese compared to lean T2DM patients, but that it is also linked to cardiac contractile dysfunction and fibroinflammatory liver disease. However, myocardial steatosis is not associated with epicardial/hepatic adiposity, and may thus represent a separate entity influenced by factors beyond ectopic adiposity. Ectopic adiposity represents an important therapeutic target, and the reversal of body fat distribution 


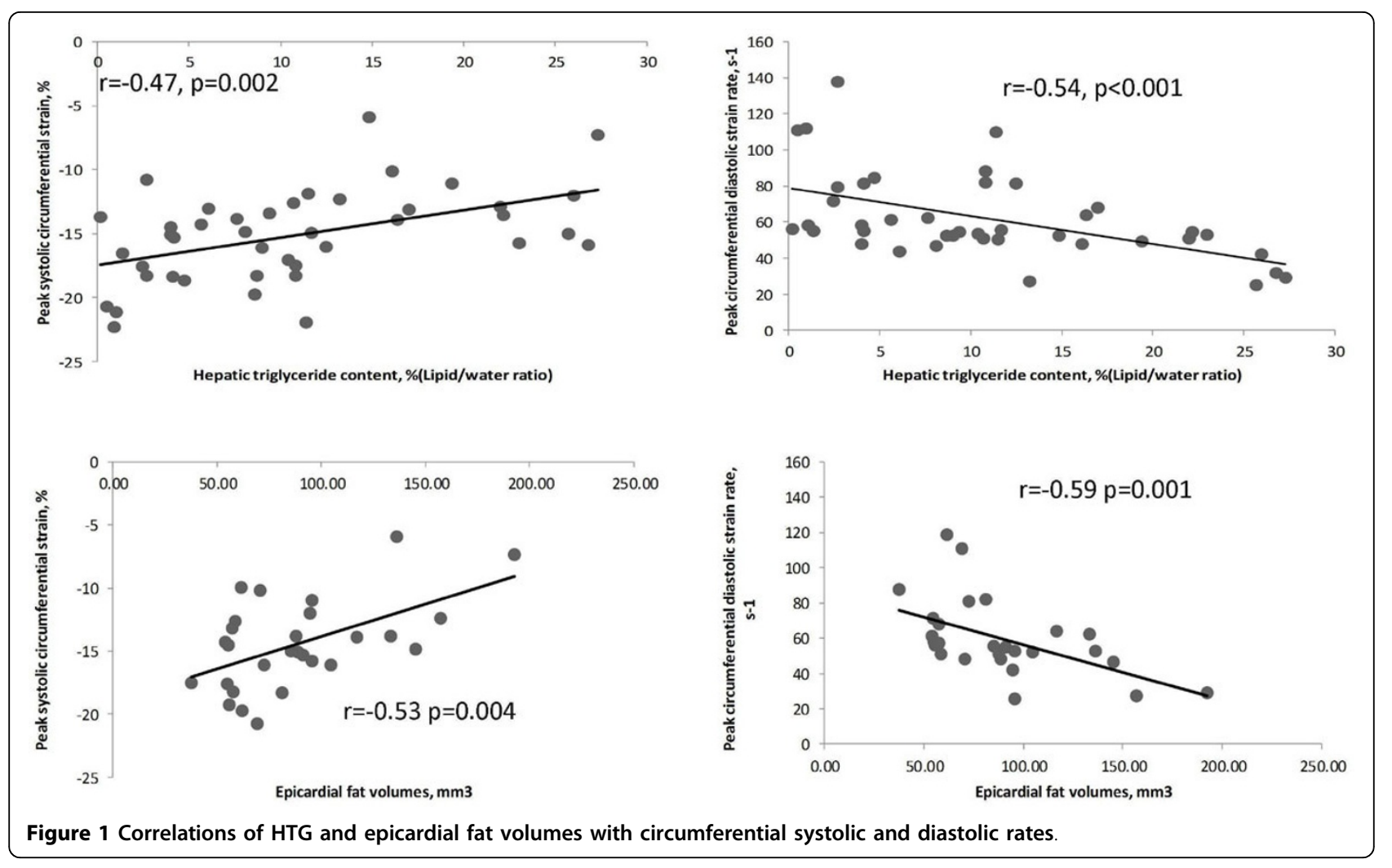

Table 1 Demographic, biochemical and multiparametric MRI results.

\begin{tabular}{|c|c|c|c|c|}
\hline & Controls $\mathrm{N}=12$ & Lean T2DM patients $\mathrm{N}=15$ & Obese T2DM patients $\mathrm{N}=\mathbf{2 7}$ & $P$ value \\
\hline Age, y & $50 \pm 10$ & $56 \pm 9$ & $56 \pm 8$ & 0.16 \\
\hline $\mathrm{BMI}, \mathrm{kg} / \mathrm{m}^{2}$ & $23 \pm 3$ & $23 \pm 2$ & $33 \pm 3^{*}$ & $<0.001$ \\
\hline Male, $\%$ & 58 & 60 & 41 & 0.41 \\
\hline Diabetes Duration, years & $\ldots$ & $6.1 \pm 4.7$ & $6.6 \pm 6.5$ & 0.78 \\
\hline Glycated hemoglobin, \% & $\ldots$ & $7.4 \pm 0.9$ & $7.7 \pm 1.4$ & 0.22 \\
\hline $\mathrm{ALT}, \mathrm{IU} / \mathrm{L}$ & $22 \pm 9$ & $30 \pm 22$ & $36 \pm 17$ & 0.12 \\
\hline \multicolumn{5}{|c|}{ Multiparametric Liver MRI } \\
\hline $\mathrm{cT1} 1 \mathrm{~ms}$ & $753 \pm 45$ & $821 \pm 67$ & $924 \pm 116^{*}$ & $<0.001$ \\
\hline Hepatic triglyceride content, \% (Lipid/water ratio) & $3.6 \pm 3.5$ & $7.7 \pm 4.6$ & $14.8 \pm 8.4^{*}$ & $<0.001$ \\
\hline \multicolumn{5}{|c|}{ CMR and Cardiac MRS Findings } \\
\hline LV end-diastolic volume, ml & $145 \pm 40$ & $124 \pm 33$ & $126 \pm 25$ & 0.15 \\
\hline LV mass, g & $98 \pm 26$ & $123 \pm 33 \dagger$ & $119 \pm 28+$ & 0.01 \\
\hline LV mass to LV end-diastolic volume, g/ml & $0.63 \pm 0.13$ & $0.95 \pm 0.26$ & $0.89 \pm 0.20$ & $<0.001$ \\
\hline Peak systolic circumferential strain, negative (-),\% & $18.1 \pm 2.1$ & $16.5 \pm 2.6 \dagger$ & $13.4 \pm 3.6^{*}$ & $<0.001$ \\
\hline Peak circumferential diastolic strain rate, s-1 & $74 \pm 20$ & $68 \pm 19^{*}$ & $56 \pm 26+$ & 0.006 \\
\hline Mycocardial PCr/ATP ratio & $2.08 \pm 0.40$ & $1.75 \pm 0.29^{*}$ & $1.64 \pm 0.32^{*}$ & 0.003 \\
\hline Mycocardial triglyceride, \%(Lipid/water ratio) & $0.48 \pm 0.28$ & $1.14 \pm 0.66+$ & $1.22 \pm 0.91 \dagger$ & 0.02 \\
\hline
\end{tabular}

Values are mean \pm standard deviations or percentages. T2DM indicates type 2 diabetes mellitus; CMR, cardiac magnetic resonancel cT1, corrected T1; ms, milliseconds; $\mathrm{PCr}$, Phs phocreatine ${ }^{*} \mathrm{p}<0.05$ vs controls and lean T2Dm $\mathrm{tp}<0.05$ vs controls 
abnormalities may improve cardiac function and prognosis in patients with diabetes.

\section{Authors' details}

'OCMR, University of Oxford, Oxford, United Kingdom. ${ }^{2}$ Perspectum Diagnostics Ltd, Oxford, United Kingdom. ${ }^{3}$ Division of Cardiovascular Medicine, John Radcliffe Hospital, Oxford, United Kingdom,. ${ }^{4}$ Department of Physiology, Anatomy \& Genetics, University of Oxford, Oxford, United Kingdom.

Published: 27 January 2016

doi:10.1186/1532-429X-18-S1-P224

Cite this article as: Levelt et al.: Cardiac and hepatic phenotype of

diabetes in the presence and the absence of obesity - mechanistic role of ectopic/visceral adiposity. Journal of Cardiovascular Magnetic Resonance 2016 18(Suppl 1):P224.

Submit your next manuscript to BioMed Central and take full advantage of:

- Convenient online submission

- Thorough peer review

- No space constraints or color figure charges

- Immediate publication on acceptance

- Inclusion in PubMed, CAS, Scopus and Google Scholar

- Research which is freely available for redistribution

Submit your manuscript at www.biomedcentral.com/submit 